\title{
The expression of Nesprin-1 increased in aortic dissection: why?
}

\author{
Wengang Yang ${ }^{1 \#}$, Dezhong Wen ${ }^{1 \#}$, Shaoxi Chen ${ }^{2}$, Song Xue ${ }^{1}$, Jianmin Gu ${ }^{1}$, Jianggui Dan ${ }^{1}$, Hui Zheng ${ }^{1}$ \\ ${ }^{1}$ Department of Cardiovascular Surgery, Ren Ji Hospital, School of Medicine, Shanghai Jiao Tong University, Shanghai 200127, China; ${ }^{2}$ Department \\ of Cardiovascular Surgery, Zhe Jiang Hospital, Hangzhou 310030, China \\ Contributions: (I) Conception and design: S Xue; (II) Administrative support: J Gu; (III) Provision of study materials and patient samples: W Yang; (IV) \\ Collection and assembly of data: J Dan, H Zheng; (V) Data analysis and interpretation: D Wen, S Chen; (VI) Manuscript writing: All authors; (VII) \\ Final approval of the manuscript: All authors. \\ \#These authors contributed equally to this work. \\ Correspondence to: Professor Song Xue; Jianmin Gu. Department of Cardiovascular Surgery, Ren Ji Hospital, School of Medicine, Shanghai Jiao Tong \\ University, Shanghai 200127, China. Email: xuesong@renji.com; gujianmin@renji.com.
}

\begin{abstract}
Background: To detect the expression of Nesprin-1 in aortic dissection (AD) in patients and to investigate the role of Nesprin-1 in the pathogenesis of $\mathrm{AD}$ in a mouse model.

Methods: Blood and tissue specimens from AD patients were collected. The expression of Nesprin-1 in tissues from $\mathrm{AD}$ patients and non-AD patients with heart disease was studied by western blotting and quantitative real-time polymerase chain reaction (qRT-PCR). In addition, the expression and distribution of Nesprin-1 in AD and sham mice were compared in an induced AD mouse model, and detected by immunohistochemistry and qRT-PCR.

Results: Immunoblotting and qRT-PCR both showed that the expression of Nesprin-1 was significantly higher in $\mathrm{AD}$ versus control patients. An animal model of $\mathrm{AD}$ was established by continuous injection of Ang II into ApoE ${ }^{-/-}$mice. The expression of Nesprin-1 in aortic tissue of AD mice was higher than that of shamoperated mice as determined by immunohistochemistry. qRT-PCR showed that Nesprin-1 gene expression in aorta of $\mathrm{AD}$ mice was higher than that of sham-operated mice.

Conclusions: An increased expression of Nesprin-1 was associated with AD, and hence Nesprin-1 may be involved in the pathogenesis of ADs. Preliminary findings suggest that Nesprin-1 may be a therapeutic target for the treatment of $\mathrm{AD}$.
\end{abstract}

Keywords: Nesprin-1; immunohistochemistry; aortic dissection (AD); pathogenesis; mouse-model

Submitted Sep 21, 2019. Accepted for publication Nov 20, 2019.

doi: $10.21037 /$ jtd.2019.12.32

View this article at: http://dx.doi.org/10.21037/jtd.2019.12.32

\section{Introduction}

Aortic dissection $(\mathrm{AD})$ is a serious disease; its pathogenesis is unknown, and there is a lack of targeted treatment for $\mathrm{AD}$. These factors restrict the therapeutic effect. Relevant studies have shown that Nesprin-1 plays an important role in maintaining nuclear membrane stability, chromatin recombination, proliferation, and differentiation of cells (1-3). In this study, we investigated the expression of Nesprin-1 in $\mathrm{AD}$, in order to find a possible target for the treatment of $\mathrm{AD}$.

\section{Methods}

\section{Animals}

Apolipoprotein E homozygous negative $\left(\mathrm{ApoE}^{-/-}\right)$mice, provided by the Animal Laboratory Center of the Medical College of Shanghai, Jiaotong University. $\mathrm{ApoE}^{-/-}$mice are deficient in ApoE, and the $\mathrm{ApoE}^{-/-}$mouse is a hyperlipidemic mouse strain based on a C57BL/6 mouse background. Angiotensin II (AngII) infusion is the most common abdominal aortic aneurysm (AAA) model used in mice 
(4-6). This research was approved by the ethics committee of Shanghai Jiaotong University (syxk2017-0121).

\section{Specimen collection}

Blood and aortic specimens were collected from six AD patients during $\mathrm{AD}$ surgery for the study in total. Samples of perforated aorta from patients with coronary heart disease were collected whilst undergoing proximal anastomosis when performing a graft for coronary artery bypass. Tissue from these patients served as controls. Informed written consents were obtained from the families of the patients. The studies involving human samples were approved by the Ethics Committee of Ren Ji Hospital, School of Medicine, Shanghai Jiao Tong University [No. (2018)31K], and conformed to the principles outlined in the Declaration of Helsinki.

\section{Reagents and instruments}

Reagents: PBS solution, 10X EDTA repair solution, BSA (Roche), Anti-rabbit/rat-tong, REALTM EnVision+/HRP rabbit/mouse (Dako Denmark A/S), Real-time PCR kit (Abbott Biotechnology Co., Ltd. Shanghai), TRIZOL (Abbott Biotechnology Co., Ltd. Shanghai), Chloroform, Isopropanol (analytical purity), RNA solution (Abbott Biotechnology Co., Ltd. Shanghai), DEPC (Abbott Biotechnology Co., Ltd. Beijng), Primer synthesis (Shanghai Jierui), SDS sample buffer $(2 \times)$, Transfer buffer, $10 \times$ TBS, Blocking buffer, Wash buffer (1× TBST), Super-GL ECL Hypersensitive Luminescent Liquor (Abbot Biology), Lysis Buffer (Abbot Biology), PVDF transfer membrane (Millipore), HRP-conjugated goat-anti-rabbit IgG (Jackson) (antibody concentration 1:1,000), HRP-conjugate gated goat anti-mouse IgG (Jackson) (antibody concentration 1:1,000), Tubulin (ab52623, 52KD antibody concentration 1:5000), GAPDH (antibody concentration 1:10,000).

Equipment: Slicing machine (Leica Instrument Co., Ltd. Shanghai), Decolorization shaker (Liuyi Instruments, Beijing), Ordinary optical microscopy mirror (Chongqing Photoelectric Instrument Co., Ltd.), Refrigerated centrifuge (Eppendorf, centrifuge 5415R), Polymerase chain reaction (Eppendorf), Real-time fluorescence quantitative PCR (Agilent Stratagene, MX3000P). PowerPacTMHC electrophoresis (Bio-rad), VE-180 vertical electrophoresis cell (Tianneng), Semi-Dry Transfer Cell (Bio-rad), DY-B1 decolorization shaking bed (Shanghai Qingpu Shanghai). Instrumentation used for western blots (Bio-rad), KODAK X-Omat BT Film (Kodak), $\mathrm{X}$-ray developer, X-ray fixer (Kodak).

\section{Western blot}

After the patient samples were processed, $300 \mu \mathrm{L}$ of the resultant tissue cell lysate were removed and mixed with gun to complete the lysis. Thereafter the lysate was transferred to a clean centrifuge tube, and $10 \mu \mathrm{L}$ of sample was added to $10 \mu \mathrm{L}$ of $2 \times$ SDS-PAGE loading buffer. The sample and buffer were mixed, heated for 5 minutes at $100{ }^{\circ} \mathrm{C}$, cooled on ice and centrifuged for 5 minutes at to remove insoluble precipitation. The supernatant $(20 \mu \mathrm{L}$ of each sample) was loaded into each well of a $10 \%$ SDS-PAGE gel, and the proteins were separated. Following electrophoresis, a PVDF membrane was soaked in methanol for 1 minute, then soaked in transfer buffer, filter paper and PVDF film soaked in methanol for 4 minutes for 10 minutes, then the transfer sandwich was prepared. A semi-dry cell was used for semidry electrophoretic transfer at $30 \mathrm{~mA}$ for 50 minutes. Blocking buffer was used to seal the transfer film 4 unitary C overnight, and $1 \times$ TBST was used to wash the membrane three times the next day for 15 minutes each time. The membrane was incubated for 2 hours with diluted primary antibody. Thereafter the membrane was washed four times with $1 \times$ TBST for 10 minutes each time and the diluted secondary antibody was added and incubated for 2 hours. The membrane was washed four times with $1 \times$ TBST for 10 minutes each time. Super-GL ECL supersensitive luminescent reagent was used for chemiluminescence detection and $\mathrm{X}$-ray exposure. After fixing, the air-dried film was photographed with a gel imaging analysis system and analyzed using Gel-Pro Analyzer version 4.0 software (Media Cybernetics, Inc., Rockville, MD, USA).

\section{Quantitative real-time polymerase chain reaction (RT- PCR)}

RNA extraction: Tissue samples were treated, cells were collected, and $1 \mathrm{~mL}$ Trizol was added. Chloroform $(200 \mu \mathrm{L})$ was added to the Trizol-sample and emulsified at room temperature for 5 minutes. Following centrifugation at $12,000 \mathrm{~g}$ at $4{ }^{\circ} \mathrm{C}$ for 15 minutes, the supernatant was transferred to a new $1.5 \mathrm{~mL}$ centrifuge tube and an equal volume of isopropanol was added to the supernatant, mixed, and then allowed to precipitate at room temperature for 10 minutes. The samples were then centrifuged at $12,000 \mathrm{~g}$ at $4{ }^{\circ} \mathrm{C}$ for 10 minutes. The supernatant was discarded, and $1 \mathrm{~mL}$ of $70 \%$ ethanol was added and the tube was gently inverted. The samples were centrifuged at $12,000 \mathrm{~g}$ at $4{ }^{\circ} \mathrm{C}$ for 5 minutes and the ethanol was carefully discarded. The 


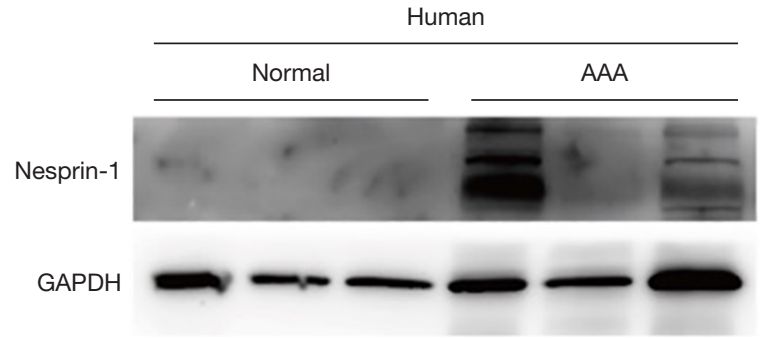

Figure $1 \mathrm{WB}$ detected the expression of Nesprin-1 in AD patients and normal control group. We collected aortic tissues of AD patients and found that the expression of Nesprin-1 increased in $\mathrm{AD}$ patients by immunoblotting, with statistical difference. It was confirmed in $\mathrm{AD}$ that the increasing trend of Nesprin-1 expression indicates that Nesprin-1 protein is involved in the pathogenesis of AD. This result is of great significance. Nesprin-1 has nuclear membrane and organelles, and plays a key role in mitosis, stabilization of nuclear membrane, chromatin recombination and separation, and RNA replication and translocation of adult cells. The change of protein level lays a solid foundation for functional realization.

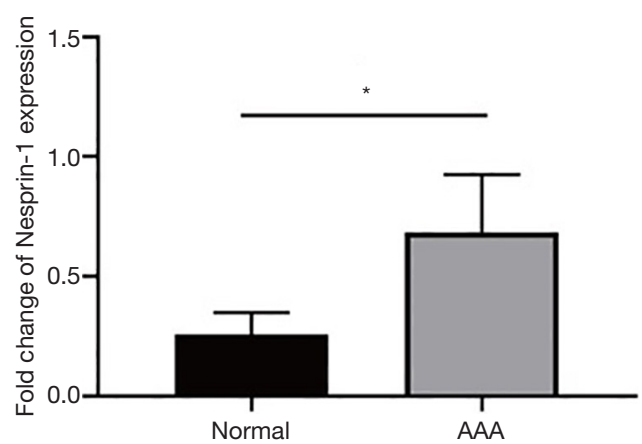

Figure 2 The expression of Nesprin-1 in $\mathrm{AD}$ patients and normal controls was detected by qRT-PCR. The expression of Nesprin-1 in $\mathrm{AD}$ patients was higher than control group detected by realtime fluorescence quantitative PCR, with statistical difference. It suggests that Nesprin-1 may be involved in $\mathrm{AD}$ at transcriptional level. It can be concluded that the change of Nesprin-1 protein level is accompanied by the regulation of transcription level. It can be inferred that the increase of Nesprin-1 protein level is due to the dual effects of transcription level and protein level.

precipitate was dried at room temperature for 2-5 minutes, and $20 \mu \mathrm{L}$ of RNA solution was added to dissolve the precipitate on ice for approximately 30 minutes.

Total RNA reverse transcription and RT-PCR: RNA reverse transcription, real-time PCR: a two-step method with GAPDH as the normalization gene: primer 5 software was used to design the primers, and they were then synthesized by Abbott biological design. Relative quantification was used in statistical analysis. Relative expression was calculated by RQ $=2$-Delta CT. Excel software was used for data analysis and for drawing the graphs.

\section{Immunobistochemistry}

Paraffin embedded tissue sections were baked at $65{ }^{\circ} \mathrm{C}$ for 2 hours, dewaxed, and rinsed with PBS three times, for 5 minutes each time. Antigen retrieval of the sections was performed by microwaving in EDTA buffer solution. The power was cut off from mid-fire to boiling, and low-fire to boiling at intervals of 10 minutes. After natural cooling, the sections were washed three times with PBS for 5 minutes each time. Sections were incubated at room temperature for 10 minutes in $3 \%$ hydrogen peroxide solution to block endogenous peroxidase. The sections were washed three times with PBS for 5 minutes each time and 5\% BSA was added to the sections and the vessel sealed for 20 minutes. BSA solution was removed and $50 \mathrm{~mL}$ diluted primary antibody was added to each section and left overnight at 4 ${ }^{\circ} \mathrm{C}$. The sections were once again washed three times with PBS for 5 minutes each time. The PBS was discarded and the secondary antibody of the corresponding species was added to each section, and incubated at $4^{\circ} \mathrm{C}$ for 50 minutes. The sections were washed three times with PBS, for 5 minutes each time. The PBS solution was discarded and 50-100 mL freshly prepared DAB solution was adding to each section. After completing the color reaction, the sections were rinsed with distilled or tap water, re-stained with hematoxylin, 1\% hydrochloric acid alcohol differentiation (1 s), rinsed with tap water, ammonia water, and finally rinsed with running water. The slices were dehydrated and dried with gradient alcohol (70-100\%) for 10 minutes, coated with xylene and sealed with neutral gum.

\section{Results}

Western blot was used to detect the expression of Nesprin-1 in $\mathrm{AD}$ patients and in a non-AD control group with heart disease. This finding was confirmed by immunoblotting $(\mathrm{P}<0.05)$ as demonstrated in Figure 1.

The expression of Nesprin-1 in tissues from AD patients was statistically higher than that of the control group $(\mathrm{P}<0.05)$. These findings suggest that Nesprin-1 may be involved in $\mathrm{AD}$ at a transcriptional level (Figure 2). 


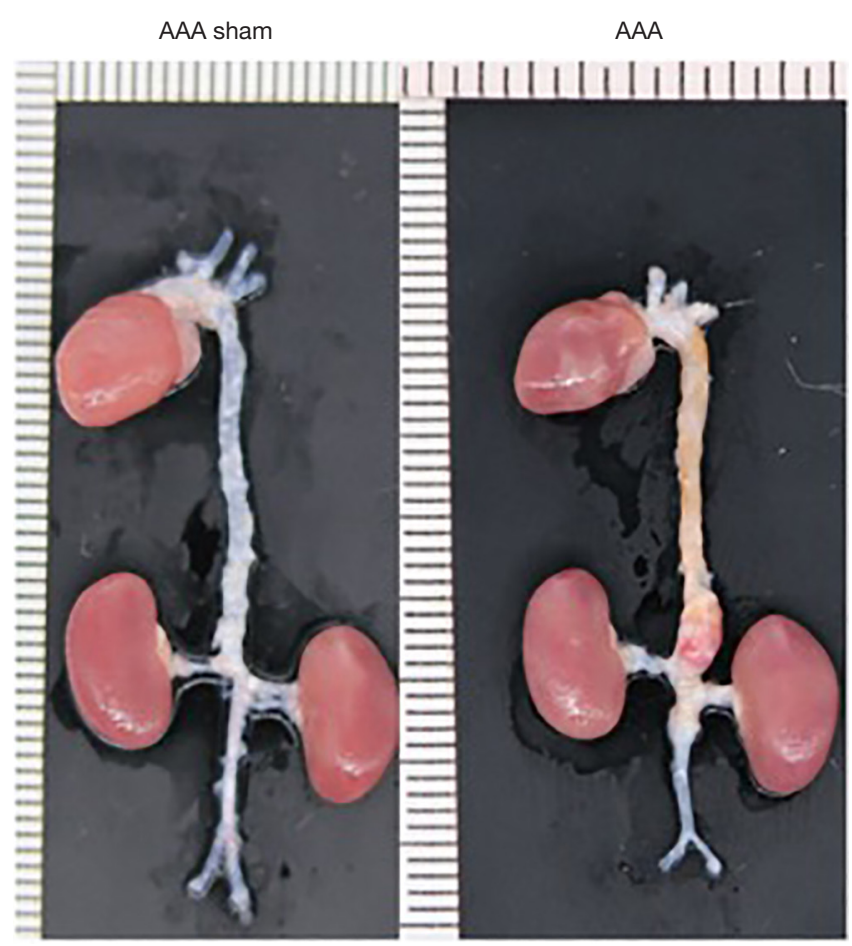

Figure 3 The AD animal model was successfully established by continuous injection of Ang II into $\mathrm{ApoE}^{-/-}$mice through subcutaneous embedding micropump. Animal models play an important role in the study of $\mathrm{AD}$ mechanism.

An AD animal model was successfully established by continuous injection of Ang II into $\mathrm{ApoE}^{-/-}$mice by using a subcutaneously embedded micropump (Figure 3).

Immunohistochemical detection of Nesprin-1 distribution and expression in AD animal model: immunohistochemical detection showed that Nesprin-1 expression in aortic tissue of $\mathrm{AD}$ mice was higher than that detected in the aortic tissue of the sham operation group. This indicates that Nesprin-1 may be involved in the pathogenesis of $\mathrm{AD}$ (Figure 4).

The expression of Nesprin-1 in the aorta of AD mice was detected by qRT-PCR. qRT-PCR showed that the expression of Nesprin-1 in the aorta of $\mathrm{AD}$ mice was higher than that of sham-operated mice (Figure 5).

\section{Discussion}

$\mathrm{AD}$ is a serious disease with a high mortality rate (7). At the onset of the disease, the blood in the aortic cavity enters the media from the intimal fissure, which separates the media and expands along the long axis of the aorta,

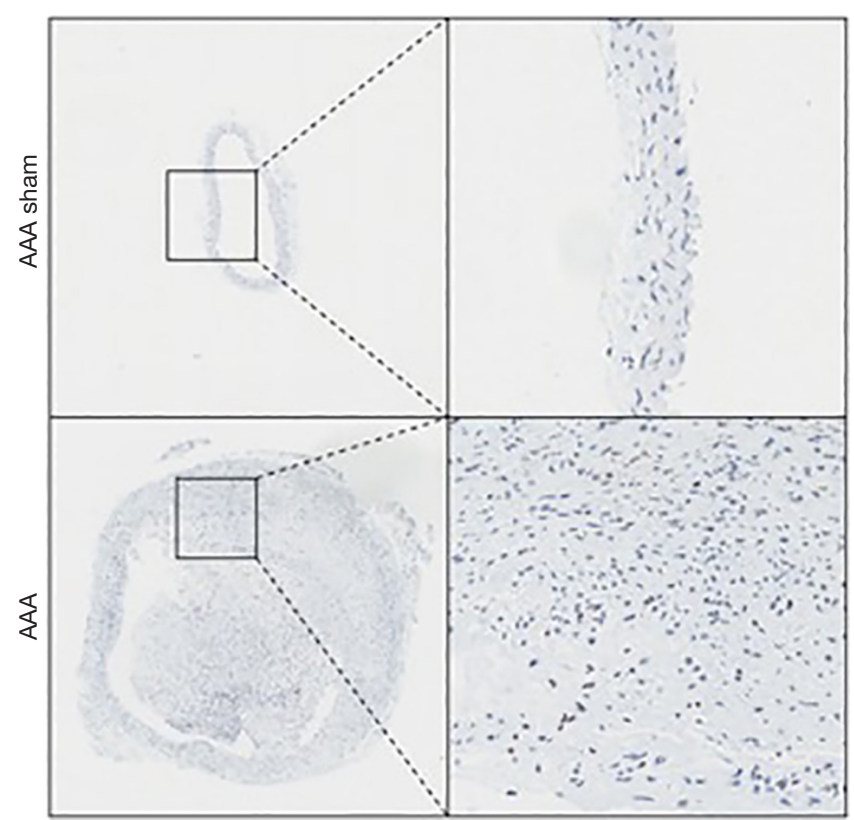

Figure 4 The expression and distribution of Nesprin-1 were detected by immunohistochemical. Compared with sham-operated group, the expression of Nesprin-1 in aorta tissue of AD mice was increasing. This indicates that Nesprin-1 is involving in the pathogenesis of $\mathrm{AD}$.

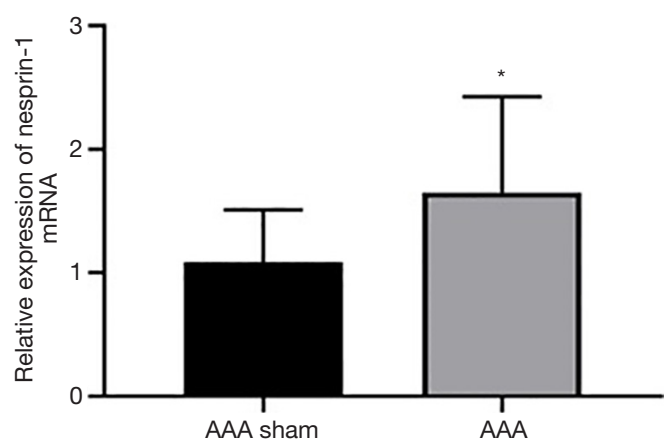

Figure 5 The expression of Nesprin-1 in aortic tissue of AD mice was detected by real-time fluorescence quantitative PCR. Compared with sham-operated group, the expression of Nesprin-1 in aortic tissue of $\mathrm{AD}$ mice was increasing. It suggests that Nesprin-1 may be involved in $\mathrm{AD}$ at transcriptional level. *, $\mathrm{P}<0.05$.

forming the separation state of the aortic wall. Acute $\mathrm{AD}$ manifests as an intimal tear, with blood entering the denatured elastic media, resulting in a loss of smooth muscle cells. This results in endometrial hyperplasia, apoptosis of smooth muscle cells and rupture of elastic fibers in aorta of patients with chronic AD. Many cytokines, 
proteases and related signaling pathways are involved in the occurrence of $\mathrm{AD}$, and the absence of TGF-beta leads to the enhancement of the inflammatory response pathways (8). Hypertension is an important risk factor for AD. The study of chronic hypertension in an animal model found that the blood flow of aortic trophoblast decreased, which led to the abnormal morphology of elastin and collagen fibers in the adventitia, necrosis of the media and vascular degeneration (9), and ultimately it promoted the occurrence of $\mathrm{AD}(10)$. We successfully established an $\mathrm{AD}$ animal model by continuous injection of Ang II into $\mathrm{ApoE}^{-/-}$mice by using a subcutaneously embedded micropump.

Despite the association with hypertension, the pathogenesis of $\mathrm{AD}$ remains unknown and there are no targeted drugs, thus restricting the therapeutic effect of treatment options. Therefore, there is an urgent need to clarify the pathogenesis of $\mathrm{AD}$ and identify relevant therapeutic targets to improve disease outcome.

In this study, we collected aortic samples from $\mathrm{AD}$ patients and established a model of $\mathrm{AD}$ artery. We found that the increasing trend of Nesprin-1 expression in $\mathrm{AD}$ indicated that Nesprin-1 protein was involved in the pathogenesis of AD. In addition, the expression of Nesprin-1 mRNA was increased in the aorta of mice with induced $\mathrm{AD}$, further supporting the association between $\mathrm{AD}$ and increased Nesprin-1 expression.

Nesprin-1 is expressed in human tissues and organs, particularly in the spleen, peripheral blood leukocytes and heart. Overexpression of Nesprin-1 results in morphological changes in the Golgi apparatus and Nesprin-1 is important for the maintaining Golgi structure. Stability plays an important role in the Golgi bodies. Nesprin-1 plays a role in the transport of substances in Golgi and endoplasmic reticulum (11). The KASH (Klarsicht/ANC-1/Syne homologue) region in Nesprins-1 interacts with SUN-1domain in the intercellular space of the cell membrane. At the same time, the KASH region of proteins in the perinuclear space interacts with the SUN region to form a LINC complex, which exists between the nucleus and the cytoskeleton. Through the bridging of the LINC (Nucleoskeleton-and-Cytoskeleton) complex and cytoskeleton actin, the mechanism of maintaining the nuclear membrane is preserved (1). Experiments also showed that Nesprin-1 protein was linked to F-actin $(2,3)$, and played a role in nuclear separation and migration $(2,12)$. Nesprin-1 protein binds to actin in the nucleus of adult cells, participates in the modification process of actin, plays an important role in the nuclear membrane, and participates in the transcriptional control of chromatin remodeling $(13,14)$. Nesprin-1 transfers from the nucleus and nuclear membrane to the cytoplasm and sarcomere during the differentiation of $\mathrm{C} 2 \mathrm{C} 12$ muscle cells into myotubes, suggesting that Nesprin-1 plays an important and unique role in muscle differentiation.

Smith et al. interfered with Nesprin-1 expression by manipulating siRNA. During the differentiation of embryonic stem cells, it was found that the nuclear membrane of differentiated cells arranged more regularly and tightly than that of pre-differentiated cells. It was suggested that the change of nuclear membrane structure accompanied with the process of cell differentiation might be involved in promoting the staining of differentiated cells. It may be involved in promoting chromatin recombination of differentiated cells (15). In conclusion, Nesprin-1 exists in the nuclear membrane and organelles, and plays a key role in mitosis of adult cells, stabilization of nuclear membranes, chromatin recombination and separation, and RNA replication and transport.

In this study, we found that the expression of Nesprin-1 was elevated in $\mathrm{AD}$ patients and in the aorta of $\mathrm{AD}$ mice. This led us to investigate whether there was a change in Nesprin-1 protein expression. Previously, we mentioned that hypertension is an important cause of $\mathrm{AD}$, but not all patients with hypertension suffer from $\mathrm{AD}$. It is speculated that the effect of hypertension on the aortic wall disturbs the stability of the cells. In order to maintain the stability of the cell skeleton, cells secrete a large amount of Nesprin-1 to maintain the rigid structure. If the rigid structure cannot be maintained, the cells and the cell junctions will rupture, resulting in endovascular rupture of $\mathrm{AD}$. For this reason, we investigated the change in expression of Nesprin-1 in patient specimens and in an animal model, and found the expression to be increased.

Nesprin-1 may be a target for predicting the occurrence and guiding the treatment of AD. This study lays a solid foundation for further research on its downstream mechanisms and treatment directions. In this study, we established an $\mathrm{AD}$ animal model to strengthen our conclusions. We have verified the consistency between the findings from $\mathrm{AD}$ in humans and in an $\mathrm{AD}$ mouse model. The establishment of the $\mathrm{AD}$ mouse model will provide an opportunity for further studies for investigating the mechanism of Nesprin-1 activity on the formation of aortic aneurysm, and provide a theoretical basis for the clinical intervention of aortic aneurysm and $\mathrm{AD}$. 


\section{Acknowledgments}

Funding: This study was supported by a grant from the Wu Jieping Medical Fund, China (No. 320.6750.17557) and the Shenkang specialty disease clinical "five new" transformation project (16CR3086B).

\section{Footnote}

Conflicts of Interest: The authors have no conflicts of interest to declare.

Ethical Statement: The authors are accountable for all aspects of the work in ensuring that questions related to the accuracy or integrity of any part of the work are appropriately investigated and resolved. The institutional review board of Ren Ji Hospital, Shanghai Jiao Tong University School of Medicine, approved the use of a prospectively maintained database of patients with symptomatic $\mathrm{AD}$ and non-AD patients with coronary heart disease for this study [No. (2018)31K].

\section{References}

1. Lei K, Zhang X, Ding X, et al. SUN1 and SUN2 play critical but partially redundant roles in anchoring nuclei in skeletal muscle cells in mice. Proc Natl Acad Sci U S A 2009; 106:10207-12.

2. Zhen YY, Libotte T, Munck M, et al. NUANCE, a giant protein connecting the nucleus and actin cytoskeleton. J Cell Sci 2002;115:3207-22.

3. Padmakumar VC, Abraham S, Braune S, et al. Enaptin, a giant actin-binding protein, is an element of the nuclear membrane and the actin cytoskeleton. Exp Cell Res 2004;295:330-9.

4. Rush C, Nyara M, Moxon JV, et al. Whole genome expression analysis within the angiotensin IIapolipoprotein $\mathrm{E}$ deficient mouse model of abdominal aortic aneurysm. BMC Genomics 2009;10:298.

5. Inoue N, Muramatsu M, Jin D, et al. Involvement

Cite this article as: Yang W, Wen D, Chen S, Xue S, Gu J, Dan J, Zheng H. The expression of Nesprin-1 increased in aortic dissection: why? J Thorac Dis 2019;11(12):4960-4965. doi: $10.21037 /$ jtd.2019.12.32 of vascular angiotensin II-forming enzymes in the progression of aortic abdominal aneurysms in angiotensin II- infused ApoE-deficient mice. J Atheroscler Thromb 2009;16:164-71.

6. Zhang Z, Zou G, Chen X, et al. Knockdown of lncRNA PVT1 Inhibits Vascular Smooth Muscle Cell Apoptosis and Extracellular Matrix Disruption in a Murine Abdominal Aortic Aneurysm Model. Mol Cells 2019;42:218-27.

7. Mészáros I, Mórocz J, Szlávi J, et al. Epidemiology and clinicopathology of aortic dissection. Chest 2000;117:1271-8.

8. Bondy CA. Aortic dissection in Turner syndrome. Curr Opin Cardiol 2008;23:519-26.

9. Baikoussis NG, Apostolakis EE, Papakonstantinou NA, et al. The implication of vasa vasorum in surgical diseases of the aorta. Eur J Cardiothorac Surg 2011;40:412-7.

10. Marcus ML, Heistad DD, Armstrong ML, et al. Effects of chronic hypertension on vasa vasorum in the thoracic aorta. Cardiovasc Res 1985;19:777-81.

11. Kobayashi Y, Katanosaka Y, Iwata Y, et al. Identification and characterization of GSRP-56, a novel Golgilocalized spectrin repeat-containing protein. Exp Cell Res 2006;312:3152-64.

12. Williams AR, Hare JM. Mesenchymal stem cells: biology, pathophysiology, translational findings, and therapeutic implications for cardiac disease. Circ Res 2011;109:923-40.

13. Zhang Q, Skepper JN, Yang F, et al. Nesprins: a novel family of spectrin-repeat-containing proteins that localize to the nuclear membrane in multiple tissues. J Cell Sci 2001;114:4485-98.

14. Zhang Q, Ragnauth CD, Skepper JN, et al. Nesprin-2 is a multi-isomeric protein that binds lamin and emerin at the nuclear envelope and forms a subcellular network in skeletal muscle. J Cell Sci 2005;118:673-87.

15. Smith ER, Zhang XY, Capo-Chichi CD, et al. Increased expression of Syne1/nesprin-1 facilitates nuclear envelope structure changes in embryonic stem cell differentiation. Dev Dyn 2011;240:2245-55. 